PROCEEDINGS OF THE

AMERICAN MATHEMATICAL SOCIETY

Volume 137, Number 9, September 2009, Pages 3003-3011

S 0002-9939(09)09898-0

Article electronically published on April 10, 2009

\title{
LARGE-TIME BEHAVIOR OF PERIODIC ENTROPY SOLUTIONS TO ANISOTROPIC DEGENERATE PARABOLIC-HYPERBOLIC EQUATIONS
}

\author{
GUI-QIANG CHEN AND BENOÎT PERTHAME
}

(Communicated by Walter Craig)

\begin{abstract}
We are interested in the large-time behavior of periodic entropy solutions in $L^{\infty}$ to anisotropic degenerate parabolic-hyperbolic equations of second order. Unlike the pure hyperbolic case, the nonlinear equation is no longer self-similar invariant, and the diffusion term in the equation significantly affects the large-time behavior of solutions; thus the approach developed earlier, based on the self-similar scaling, does not directly apply. In this paper, we develop another approach for establishing the decay of periodic solutions for anisotropic degenerate parabolic-hyperbolic equations. The proof is based on the kinetic formulation of entropy solutions. It involves time translations and a monotonicity-in-time property of entropy solutions and employs the advantages of the precise kinetic equation for the solutions in order to recognize the role of nonlinearity-diffusivity of the equation.
\end{abstract}

\section{INTRODUCTION}

We study the large-time behavior of periodic solutions in $L^{\infty}$ to nonlinear anisotropic degenerate parabolic-hyperbolic equations of second order. Consider the Cauchy problem for the second-order equations

$$
\begin{aligned}
\partial_{t} u & +\nabla_{x} \cdot f(u)=\nabla_{x} \cdot\left(A(u) \nabla_{x} u\right), \quad x \in \mathbb{R}^{d}, \quad t \geq 0, \\
\left.u\right|_{t=0} & =u_{0} \in L^{\infty}\left(\mathbb{R}^{d}\right), \quad u_{0} \text { is } \mathbb{T}_{P} \text {-periodic, }
\end{aligned}
$$

where $\mathbb{T}_{P}:=\prod_{i=1}^{d}\left[0, P_{i}\right]$, with $P_{i}>0$, and by " $\mathbb{T}_{P}$-periodic" we mean $u\left(x+P_{i} e_{i}\right)=$ $u(x)$ with $\left(e_{i}\right)_{1 \leq i \leq d}$ the canonic basis of $\mathbb{R}^{d}$. The flux function $f: \mathbb{R} \rightarrow \mathbb{R}^{d}$ satisfies

$$
a(\cdot):=f^{\prime}(\cdot) \in L_{\mathrm{loc}}^{\infty}\left(\mathbb{R} ; \mathbb{R}^{d}\right),
$$

Received by the editors October 16, 2008.

2000 Mathematics Subject Classification. Primary 35K65, 35K15, 35B10, 35B40, 35D99; Secondary 35K10, 35B30, 35B41, 35M10, 35L65.

Key words and phrases. Periodic solutions, entropy solutions, decay, large-time behavior, kinetic formulation, degenerate parabolic equations, anisotropic diffusion, nonlinearity-diffusivity.

The first author's research was supported in part by the National Science Foundation under Grants DMS-0244473, DMS-0807551, DMS-0720925, and DMS-0505473; an Alexander von Humboldt Foundation Fellowship; and the Natural Science Foundation of China under Grant NSFC-10728101. This paper was written as part of the International Research Program on Nonlinear Partial Differential Equations at the Centre for Advanced Study at the Norwegian Academy of Science and Letters in Oslo during the academic year 2008-09.

(C)2009 American Mathematical Society Reverts to public domain 28 years from publication 
and the $d \times d$ matrix $A(u)=\left(a_{i j}(u)\right)$ is symmetric, nonnegative, and locally bounded so that it can always be written in the form

$$
a_{i j}(u)=\sum_{k=1}^{d} \sigma_{i k}(u) \sigma_{j k}(u), \quad \sigma_{i k} \in L_{\mathrm{loc}}^{\infty}(\mathbb{R}),
$$

with $\left(\sigma_{i k}(u)\right)$ the square root matrix of $A(u)$.

Equation (1.1) and its variants model degenerate diffusion-convection motions of ideal fluids and arise in a wide variety of important applications (cf. [1, 2, 4, 12, 20] and the references cited therein), for which a deep understanding of solutions to (1.1) is in great demand, at both the theoretical and numerical levels.

In Chen-Perthame 7, a well-posedness theory has been established for $L^{1}$ solutions of the Cauchy problem (1.1) and (1.2) of anisotropic degenerate parabolichyperbolic equations of second order. It extends the isotropic theory for degenerate parabolic-hyperbolic equations and several later studies; see for instance [3, 5, 16, 18, and the references therein. A notion of kinetic solutions, a new concept in this context, and a corresponding kinetic formulation have been extended. In particular, it has also been proved that, when $u_{0} \in L^{\infty}$, the kinetic solution is equivalent to the entropy solution, or to the dissipative solution 23, which is unique; this provides a new path to study the behavior of entropy solutions in $L^{\infty}$ through the corresponding kinetic equations for anisotropic degenerate parabolichyperbolic equations. In this paper, we employ the advantages of this path to develop a new approach for establishing the decay of periodic entropy solutions in $L^{\infty}$ to (1.1) and (1.2) as $t \rightarrow \infty$. The main theorem of this paper is the following.

Theorem 1.1 (Main theorem). Assume (1.3) and (1.4) hold. Let $u \in L^{\infty}([0, \infty) \times$ $\left.\mathbb{R}^{d}\right)$ be the unique periodic entropy solution to (1.1) and (1.2). Assume that the flux function and the diffusion matrix $A(u)$ satisfy the nonlinearity-diffusivity condition: For any $\delta>0$,

$$
\sup _{|\tau|+|\kappa| \geq \delta} \int_{|\xi| \leq\left\|u^{0}\right\|_{\infty}} \frac{\lambda d \xi}{\lambda+|\tau+a(\xi) \cdot \kappa|^{2}+\left(\kappa^{\top} A(\xi) \kappa\right)^{2}}:=\omega_{\delta}(\lambda) \underset{\lambda \rightarrow 0}{\longrightarrow} 0 .
$$

Then we have

$$
\|u(t, \cdot)-\bar{u}\|_{L^{1}\left(\mathbb{T}_{P}\right)} \underset{t \rightarrow \infty}{\longrightarrow} 0
$$

where

$$
\bar{u}=\frac{1}{\left|\mathbb{T}_{P}\right|} \int_{\mathbb{T}_{P}} u_{0}(x) d x .
$$

The nonlinearity-diffusivity condition (1.5) for equation (1.1) is developed from [13, 17] and is reminiscent of the theory of velocity averaging lemmas [13, 21, 22, 25]. For smooth coefficients, condition (1.5) is equivalent to the simpler and more standard setting: For any $(\tau, \kappa) \in \mathbb{R}^{d+1}$ with $\tau^{2}+|\kappa|^{2}=1$, we have

$$
\mathcal{L}^{1}\left\{\xi \in \mathbb{R}:|\xi| \leq\left\|u_{0}\right\|_{\infty}, \tau+a(\xi) \cdot \kappa=0, \kappa^{\top} A(\xi) \kappa=0\right\}=0 .
$$

Several explicit examples are given in [17, 25]. This condition implies that there is no interval of $\xi$ on which both the flux function $f(\xi)$ is affine and the diffusion matrix $A(\xi)$ is degenerate, and thus it also makes the relation with the applications of the theory of compensated compactness [19, 26] to one-dimensional hyperbolic conservation laws. Such a nonlinearity-diffusivity condition is necessary for the 
decay of periodic solutions and the compactness of solution operators. See also the books [8, 24].

Theorem 1.1 also extends naturally the nondegeneracy condition for the purely hyperbolic case in 11, where the first long-time convergence result of periodic solutions was obtained in one or two dimensions for $B V$ initial data and higher order local nondegeneracy conditions that replace (1.5).

Unlike the pure hyperbolic case, equation (1.1) is no longer self-similar invariant, and the diffusion term in the equation significantly affects the large-time behavior of solutions; thus the approach in [6] based on the self-similar scaling for the pure hyperbolic case does not apply, and we have to change the strategy of proof. The approach developed in this paper is based on the kinetic formulation of entropy solutions in 7, involves time translations and a monotonicity-in-time of entropy solutions, and employs the advantages of the kinetic equations of the solutions in order to recognize the role of the nonlinearity-diffusivity of the equation.

The rest of this paper is organized as follows. We first recall the notion of entropy solutions and their precise kinetic formulation and then analyze some basic properties of entropy solutions. Finally, we develop a new approach to give a rigorous proof for the long-time asymptotic result.

\section{ENTROPY SOLUTIONS AND KINETIC FORMULATION}

In this section, we first recall the notion of entropy solutions and their precise kinetic formulation, which requires some care to define appropriately the various terms of the equation. Then we analyze some basic properties of entropy solutions, which will be used in the proof of Theorem 1.1

Definition 2.1. An entropy solution is a function $u(t, x) \in L^{\infty}\left([0, \infty) \times \mathbb{R}^{d}\right)$ such that

(i) $\sum_{i=1}^{d} \partial_{x_{i}} \beta_{i k}(u) \in L^{2}\left([0, \infty) \times \mathbb{R}^{d}\right), k=1, \cdots, d$, for $\beta_{i k}(u)=\int^{u} \sigma_{i k}(v) d v$.

(ii) For any function $\psi \in C_{0}(\mathbb{R})$ with $\psi(u) \geq 0$ and any $k=1, \cdots, d$, the chain rule holds:

$$
\sum_{i=1}^{d} \partial_{x_{i}} \beta_{i k}^{\psi}(u)=\sqrt{\psi(u)} \sum_{i=1}^{d} \partial_{x_{i}} \beta_{i k}(u) \in L^{2}\left([0, \infty) \times \mathbb{R}^{d}\right) \quad \text { for }\left(\beta_{i k}^{\psi}\right)^{\prime}=\sqrt{\psi} \beta_{i k}^{\prime} .
$$

(iii) For any smooth function $S(u)$, there exists an entropy dissipation measure $m^{S^{\prime \prime}}(t, x)$ satisfying

$$
m^{S^{\prime \prime}}(t, x)=\int_{\mathbb{R}} S^{\prime \prime}(\xi) m(t, x, \xi) d \xi \quad \text { with } m(t, x, \xi) \text { a nonnegative measure, }
$$

such that

$$
\partial_{t} S(u)+\nabla_{x} \cdot q^{S}(u)-\nabla_{x} \cdot\left(A(\xi) \nabla_{x} S(u)\right)=-\left(m^{S^{\prime \prime}}+n^{S^{\prime \prime}}\right)
$$

in $\mathcal{D}^{\prime}\left(\mathbb{R}^{+} \times \mathbb{R}^{d}\right)$ with initial data $\left.S(u)\right|_{t=0}=S\left(u_{0}\right)$, where $q^{S}: \mathbb{R} \rightarrow \mathbb{R}^{d}$ is the corresponding entropy flux, i.e. $\left(q^{S}(u)\right)^{\prime}=S^{\prime}(u) f^{\prime}(u)$, and

$$
n^{S^{\prime \prime}}(t, x, \xi)=\int S^{\prime \prime}(\xi) n(t, x, \xi) d \xi
$$


for $n(t, x, \xi)$ the parabolic defect measure of $u(t, x)$ defined as

$$
n(t, x, \xi):=\delta(\xi-u(t, x)) \sum_{k=1}^{d}\left(\sum_{i=1}^{d} \partial_{x_{i}} \beta_{i k}(u(t, x))\right)^{2} \quad \text { a.e. }
$$

We point out that the chain rule in (ii) has to be assumed in the anisotropic case, and this makes the main difference with the isotropic case in [3, where this property follows from an argument reminiscent of the theory of Sobolev spaces. Requirement (iii) has been made with notation that is adapted to the kinetic formulation we introduce now. The $L^{2}$-condition in (i) is required to define the parabolic defect measure $n(t, x, \xi)$ in (2.4) (also see [5]).

To do so, we may factor out an $S^{\prime}(u)$ in equation (2.3) and obtain a useful kinetic formulation of nonlinear degenerate parabolic-hyperbolic equations of second order with form (1.1). The new ingredient of this formulation is the identification of the kinetic defect measure $m(t, x, \xi)$ and the degenerate parabolic defect measure $n(t, x, \xi)$ in a precise manner, even in the region where $u(t, x)$ is discontinuous. Compare this with the classical kinetic formulation for scalar hyperbolic conservation laws in [17] (see also 21]).

We introduce the kinetic function $\chi$ on $\mathbb{R}^{2}$ :

$$
\chi(\xi ; u)=\left\{\begin{array}{cl}
+1 & \text { for } 0<\xi<u \\
-1 & \text { for } u<\xi<0 \\
0 & \text { otherwise }
\end{array}\right.
$$

We notice that if $u$ is both in $L^{\infty}\left([0, \infty) \times \mathbb{R}^{d}\right)$ and $\mathbb{T}_{P}$-periodic in $x$, then $\chi(\xi ; u)$ is both in $L^{\infty}\left([0, \infty) \times \mathbb{R}^{d+1}\right)$ and $\mathbb{T}_{P}$-periodic in $x$.

The simple representation formula $S(u)=\int_{\mathbb{R}} S^{\prime}(\xi) \chi(\xi ; u) d \xi$ leads to the following kinetic equation, which is equivalent to the entropy identity (2.3):

$$
\partial_{t} \chi(\xi ; u)+a(\xi) \cdot \nabla_{x} \chi(\xi ; u)-\nabla_{x} \cdot\left(A(\xi) \nabla_{x} \chi(\xi ; u)\right)=\partial_{\xi}(m+n)(t, x, \xi)
$$

in $\mathcal{D}^{\prime}\left(\mathbb{R}^{+} \times \mathbb{R}^{d+1}\right)$ with initial data

$$
\left.\chi(\xi ; u)\right|_{t=0}=\chi\left(\xi ; u_{0}\right),
$$

where $n(t, x, \xi)$ is still defined through (2.4).

In [7, it has been proved that the entropy solutions in $L^{\infty}$ are equivalent to the kinetic solutions determined by the kinetic formulation (2.5)-(2.7). Furthermore, we have

Theorem 2.1. Assume that (1.3) and (1.4) hold. Then

(i) there exists a unique entropy solution $u \in L^{\infty}\left([0, \infty) \times \mathbb{R}^{d}\right)$ to (1.1)-(1.2) such that $u$ is $\mathbb{T}_{P}$-periodic;

(ii) the entropy solution $u(t, x)$ satisfies

(a) $\|u(t, \cdot)\|_{L^{\infty}} \leq\left\|u_{0}\right\|_{L^{\infty}}$;

(b) for any $t_{2}>t_{1}>0$,

$$
\begin{aligned}
& \int_{\mathbb{T}_{P}}\left|u\left(t_{2}, x\right)\right|^{2} d x \leq \int_{\mathbb{T}_{P}}\left|u\left(t_{1}, x\right)\right|^{2} d x \\
& \int_{\mathbb{T}_{P}}\left|u\left(t_{2}, x\right)-v\right| d x \leq \int_{\mathbb{T}_{P}}\left|u\left(t_{1}, x\right)-v\right| d x \quad \text { for any constant } v ;
\end{aligned}
$$


(iii) moreover, if the flux function $f(u)$ and the diffusion matrix $A(u)$ further satisfy the nonlinearity-diffusivity condition (1.5), then the solution operator $u(t, \cdot)=$ $S_{t} u_{0}(\cdot): L^{\infty} \rightarrow L^{1}$ is locally compact in $(t, x)$ for $t>0$.

The results (i)-(ii) in Theorem 2.1 are direct corollaries of the well-posedness results and the arguments in [7] (i.e. the standard entropy inequalities for $|u-v|$ and $u^{2}$ ) as in the hyperbolic case $[8,24$, while (iii) is a direct corollary of the kinetic averaging compactness result of [17]; also see more recent results in [15, 22, 25]. Point (iii) gives a hint at why dissipative effects result from assumption (1.5), but it is not fundamental in the proof below; one can also use the standard $L^{1}$ contraction property to conclude the usual compactness property we need.

\section{Dechy of Periodic entropy solutions: Proof of Theorem 1.1}

In this section, we develop a new approach to give a rigorous proof for the decay property of periodic solutions, which takes advantage of the precise kinetic formulation (2.5)-(2.7). Without loss of generality, we first set $\int_{\mathbb{T}_{P}} u(t, x) d x=0$; otherwise, we may replace $u(t, x)$ by $u(t, x)-\bar{u}, f(u)$ by $f(u+\bar{u})$, and $A(u)$ by $A(u+\bar{u})$ in (1.1), so that all the arguments below remain unchanged. Then we divide the proof into four steps.

1. Limit. Theorem 2.1 indicates that the periodic solution $u(t, x)$ belongs to $L^{\infty}$, bounded by $\left\|u_{0}\right\|_{L^{\infty}}$, and is compact as the solution operator. Also the function

$$
I(t):=\int_{\mathbb{T}_{P}}|u(t, x)|^{2} d x
$$

is a nonincreasing, bounded function, which implies that the following limit exists:

$$
\lim _{t \rightarrow \infty} I(t)=I(\infty)=: I_{\infty} \in[0, \infty)
$$

2. Translations. Set

$$
v^{k}(t, x):=u(t+k, x)
$$

Then we find that, for $t \geq-k$,

(i) $\left\|v^{k}(t, \cdot)\right\|_{L^{\infty}}=\|u(t+k, \cdot)\|_{L^{\infty}} \leq\left\|u_{0}\right\|_{L^{\infty}}$;

(ii) $v^{k}(t, x)$ is also a periodic entropy solution to (1.1)-(1.2);

(iii) for each $k>0, \chi\left(\xi ; v^{k}(t, x)\right)$ satisfies

(3.2) $\partial_{t} \chi\left(\xi ; v^{k}\right)+a(\xi) \cdot \nabla_{x} \chi\left(\xi ; v^{k}\right)-\nabla_{x} \cdot\left(A(\xi) \nabla_{x} \chi\left(\xi ; v^{k}\right)\right)=\partial_{\xi}\left(m^{k}+n^{k}\right)(t, x, \xi)$

in $\mathcal{D}^{\prime}\left((-k, \infty) \times \mathbb{R}^{d+1}\right)$.

By Theorem 2.1(iii) applied to $v^{k}$, there exist a subsequence $\left\{v^{k_{j}}\right\}_{j=1}^{\infty} \subset\left\{v^{k}\right\}_{k=1}^{\infty}$ and $v(t, x) \in L^{\infty}\left(\mathbb{R}^{d+1}\right)$, with $\int_{\mathbb{T}_{P}} v(t, x) d x=0$, such that

$$
v^{k_{j}}(t, x) \rightarrow v(t, x) \quad \text { a.e. }(t, x) \in \mathbb{R}^{d+1} \quad \text { as } j \rightarrow \infty .
$$

Correspondingly, we have

$$
\chi\left(\xi ; v^{k}(t, x)\right) \rightarrow \chi(\xi ; v(t, x)) \quad \text { a.e. }(t, x, \xi) \quad \text { as } j \rightarrow \infty .
$$

Furthermore, multiplying both sides of (3.2) by $\xi$ and then integrating over $(t, x, \xi) \in(-T, T) \times \mathbb{T}_{P} \times \mathbb{R}$ for any $T>0$, we obtain

$$
\int_{-T}^{T} \int_{\mathbb{T}_{P}}\left(m^{k}+n^{k}\right)(t, x, \xi) d t d x d \xi \leq \frac{1}{2}(I(k-T)-I(k+T)) \leq \frac{1}{2}\left|\mathbb{T}_{P}\right|\left\|u_{0}\right\|_{L^{\infty}}^{2} .
$$


This implies that the nonnegative measure sequence $\left(m^{k}+n^{k}\right)(t, x, \xi)$ is uniformly bounded in $k$ over $(-T, T) \times \mathbb{T}_{P} \times \mathbb{R}$, and hence there exist a subsequence $k_{j}$ and a measure $M(t, x, \xi)$ such that

$$
\left(m^{k_{j}}+n^{k_{j}}\right)(t, x, \xi) \rightarrow M(t, x, \xi) \geq 0 \quad \text { weakly in } \mathcal{M} \quad \text { as } j \rightarrow \infty .
$$

On the other hand, since $I(t)$ converges, we also have

$$
I(k-T)-I(T+k) \rightarrow 0 \quad \text { as } k \rightarrow \infty .
$$

Then we conclude from (3.3) that $M\left((-\infty, \infty) \times \mathbb{T}_{P} \times \mathbb{R}\right)=0$, which implies

$$
M\left(\mathbb{R}^{d+2}\right)=0 .
$$

Furthermore, letting $k \rightarrow \infty$ in (3.2), we conclude that $\chi(\xi ; v)$ is a $\mathbb{T}_{P}$-periodic solution in $\mathcal{D}^{\prime}\left(\mathbb{R} \times \mathbb{R}^{d+1}\right)$ of

$$
\partial_{t} \chi+a(\xi) \cdot \nabla_{x} \chi-\nabla_{x} \cdot\left(A(\xi) \nabla_{x} \chi\right)=0 .
$$

In particular, multiplying (3.6) by $\xi$ and then integrating $d x d \xi$, we have

$$
\int_{\mathbb{T}_{P}}|v(t, x)|^{2} d x=I_{\infty} \in[0, \infty), \quad \forall t \in \mathbb{R},
$$

where $I_{\infty}=I(\infty)$ is a constant, independent of $t$, determined in (3.1).

The rest of the proof consists of showing that such a function $\chi$ is very particular and is in fact constant (also see this type of "rigidity" results in 14, 10, 9]).

3. Claim. $v(t, x) \equiv 0$ a.e. for $(t, x) \in \mathbb{R} \times \mathbb{R}^{d}$.

We introduce a time truncation function $\phi(t), 0 \leq \phi(t) \leq 1$, so that $\phi \chi$ belongs to $L^{2}\left(\mathbb{R} \times \mathbb{T}_{P} \times \mathbb{R}\right)$. Then we have

$$
\partial_{t}(\phi \chi)+a(\xi) \cdot \nabla_{x}(\phi \chi)-\nabla_{x} \cdot\left(A(\xi) \nabla_{x}(\phi \chi)\right)=\chi \partial_{t} \phi \quad \text { in } \mathcal{D}^{\prime}\left(\mathbb{R}^{d+2}\right) .
$$

Next, since $\phi \chi$ and $\chi \phi_{t}$ are periodic in $x$, we take the global Fourier transform in $t \in \mathbb{R}$ and the local Fourier transform in $x \in \mathbb{T}_{P}$ to obtain $\hat{g}(\tau, \kappa ; \xi)$ for $(\phi \chi)(t, x, \xi)$ and $\hat{h}(\tau, \kappa ; \xi)$ for $\left(\chi \partial_{t} \phi\right)(t, x, \xi)$ in $L^{2}$, where the frequencies $\kappa=\left(\kappa_{1}, \cdots, \kappa_{d}\right)$ are discrete:

$$
\kappa_{i} \frac{2 \pi}{P_{i}}, \quad n=0, \pm 1, \pm 2, \cdots .
$$

That is, for example,

$$
\hat{g}(\tau, \kappa ; \xi)=\frac{1}{\left|\mathbb{T}_{P}\right|} \int_{\mathbb{R}} \int_{\mathbb{T}_{P}}(\phi \chi)(t, x, \xi) e^{-i(\tau t+\kappa \cdot x)} d t d x,
$$

so that

$$
(\phi \chi)(t, x, \xi)=\frac{1}{2 \pi} \sum_{\kappa} \int_{\mathbb{R}} \hat{g}(\tau, \kappa ; \xi) e^{i(\tau t+\kappa \cdot x)} d \tau .
$$

Taking the global Fourier transform in $t \in \mathbb{R}$ and the local Fourier transform in $x \in \mathbb{T}_{P}$ on both sides of (3.8), we obtain

$$
\left(i(\tau+a(\xi) \cdot \kappa)+\kappa^{\top} A(\xi) \kappa\right) \hat{g}=\hat{h} .
$$

Following the usual ideas from the kinetic averaging lemmas, we may introduce a free parameter $\lambda>0$ (to be chosen later on) and write

$$
\left(\sqrt{\lambda}+i(\tau+a(\xi) \cdot \kappa)+\kappa^{\top} A(\xi) \kappa\right) \hat{g}=\hat{h}+\sqrt{\lambda} \hat{g}
$$


This leads to

$$
\hat{g}=(\hat{h}+\sqrt{\lambda} \hat{g}) \frac{1}{\sqrt{\lambda}+i(\tau+a(\xi) \cdot \kappa)+\kappa^{\top} A(\xi) \kappa} .
$$

Integrating in $\xi$ and using the Cauchy-Schwarz inequality, we find

$$
|\widehat{\phi v}|^{2}(\tau, \kappa) \leq 2\left(\int_{\mathbb{R}} \hat{h}^{2} d \xi+\lambda \int_{\mathbb{R}} \hat{g}^{2} d \xi\right) \int_{\mathbb{R}}\left|\frac{1}{\sqrt{\lambda}+i(\tau+a(\xi) \cdot \kappa)+\kappa^{\top} A(\xi) \kappa}\right|^{2} d \xi
$$

Notice that the frequencies $\kappa$ are discrete and may include $\kappa=0$. In particular, when $\kappa \neq 0$, then there exists $\delta_{0}>0$ such that $|\kappa| \geq \delta_{0}$. On the other hand, since $v(t, x)$ has mean zero in $x$ over $\mathbb{T}_{P}$, we have

$$
\widehat{\phi v}(\tau, 0)=0 .
$$

The nonlinearity-diffusivity condition (1.5) gives that, when $\kappa \neq 0$, for any $\delta \in\left(0, \delta_{0}\right)$,

and thus

$$
|\widehat{\phi v}|^{2} \leq C \frac{\omega_{\delta}(\lambda)}{\lambda} \int_{\mathbb{R}} \hat{h}^{2} d \xi+C \omega_{\delta}(\lambda) \int_{\mathbb{R}}|\hat{g}|^{2} d \xi,
$$

$$
\begin{aligned}
\sum_{\kappa \neq 0} \int_{\mathbb{R}}|\widehat{\phi v}|^{2} d \tau & \leq C \frac{\omega_{\delta}(\lambda)}{\lambda} \sum_{\kappa \neq 0} \int_{\mathbb{R}^{2}} \hat{h}^{2} d \xi d \tau+C \omega_{\delta}(\lambda) \sum_{\kappa \neq 0} \int_{\mathbb{R}^{2}}|\hat{g}|^{2} d \xi d \tau \\
& \leq C \frac{\omega_{\delta}(\lambda)}{\lambda} \int_{\mathbb{R}^{2} \mathbb{T}_{P} \times \mathbb{R}}\left(\chi \phi_{t}\right)^{2} d t d x d \xi+C \omega_{\delta}(\lambda) \int_{\mathbb{R}^{2} \times \mathbb{T}_{P} \times \mathbb{R}}|\phi \chi|^{2} d t d x d \xi .
\end{aligned}
$$

This implies

$$
\int_{\mathbb{R} \times \mathbb{T}_{P}}|\phi v|^{2} d t d x \leq C \frac{\omega_{\delta}(\lambda)}{\lambda} \int_{\mathbb{R} \times \mathbb{T}_{P}}\left|\phi_{t}\right|^{2}|v| d t d x+C \omega_{\delta}(\lambda) \int_{\mathbb{R} \times \mathbb{T}_{P}}|\phi|^{2}|v| d t d x .
$$

Using (3.7) and the Cauchy-Schwartz inequality, we arrive at

$$
\begin{aligned}
I_{\infty} \int_{\mathbb{R}}|\phi|^{2} d t & \leq C \omega_{\delta}(\lambda)\left(\int_{\mathbb{T}_{P}}|v|^{2} d x\right)^{1 / 2}\left(\frac{1}{\lambda} \int_{\mathbb{R}}\left|\phi_{t}\right|^{2} d t+\int_{\mathbb{R}}|\phi|^{2} d t\right) \\
& \leq C I_{\infty}^{1 / 2} \omega_{\delta}(\lambda)\left(\frac{1}{\lambda} \int_{\mathbb{R}}\left|\phi_{t}\right|^{2} d t+\int_{\mathbb{R}}|\phi|^{2} d t\right) .
\end{aligned}
$$

First choosing $\lambda$ small and then $\int_{\mathbb{R}}\left|\phi_{t}\right|^{2} d t$ small, we conclude from (3.9) that

$$
I_{\infty}=0
$$

which implies from (3.7) that

$$
v(t, x) \equiv 0 \quad \text { a.e. }(t, x) \in \mathbb{R} \times \mathbb{R}^{d} .
$$

On the contrary, if $I_{\infty}>0$, we can choose $\lambda$ small enough so that $C \omega_{\delta}(\lambda) / I_{\infty}^{1 / 2} \leq$ $\frac{1}{2}$ and find from (3.9) that

$$
I_{\infty} \int_{\mathbb{R}}|\phi|^{2} d t \leq 2 C I_{\infty}^{1 / 2} \frac{\omega_{\delta}(\lambda)}{\lambda} \int_{\mathbb{R}}\left|\phi_{t}\right|^{2} d t
$$

It remains to choose a sequence of functions $\phi_{B}(t)=1$ for $|t| \leq B$, with $B$ a given large number and $\phi_{B}(t)=\frac{2 B-|t|}{B}$ for $B \leq|t| \leq 2 B$, and $\phi_{B}(t)=0$ for $|t| \geq 2 B$. In the above inequality, we find

$$
I_{\infty}^{1 / 2} \leq C \frac{\omega_{\delta}(\lambda)}{B^{2} \lambda}
$$


where $C>0$ is a constant independent of $B$ and $\lambda$. When $B$ tends to $\infty$, this implies that $I_{\infty}$ must vanish, which is a contradiction.

Therefore, (3.10) holds.

4. Conclusion. Then

$$
\int_{0}^{1} \int_{\mathbb{T}_{P}}\left|v^{k_{j}}(s, x)\right| d x d s \rightarrow 0 \quad \text { as } j \rightarrow \infty .
$$

Therefore, for any $T>k_{j}+1>k_{j}+s$ for $s \in(0,1)$, we employ (2.8) for the monotonicity-in-time property of the solution to obtain

$$
\int_{0}^{1} \int_{\mathbb{T}_{P}}\left|v^{k_{j}}(t, x)\right| d x d t \geq \int_{\mathbb{T}_{P}}\left|u\left(1+k_{j}, x\right)\right| d x \geq \int_{\mathbb{T}_{P}}|u(T, x)| d x .
$$

We conclude that

$$
\int_{\mathbb{T}_{P}}|u(T, x)| d x \rightarrow 0 \quad \text { as } T \rightarrow \infty .
$$

This completes the proof.

\section{REFERENCES}

[1] M. Bendahmane, M. Langlais, and M. Saad, On some anisotropic reaction-diffusion systems with $L^{1}$-data modeling the propagation of an epidemic disease, Nonlinear Anal. 54 (2003), no. 4, 617-636. MR:1983439 (2004c:35204)

[2] M. C. Bustos, F. Concha, R. Bürger, and E. M. Tory, Sedimentation and Thickening: Phenomenological Foundation and Mathematical Theory, Kluwer Academic Publishers: Dordrecht, Netherlands, 1999. MR.1747460 (2002b:76101)

[3] J. Carrillo, Entropy solutions for nonlinear degenerate problems, Arch. Rational Mech. Anal. 147 (1999), 269-361. MR1709116 (2000m:35132)

[4] G. Chavent and J. Jaffre, Mathematical Models and Finite Elements for Reservoir Simulation, North Holland: Amsterdam, 1986.

[5] G.-Q. Chen and E. DiBenedetto, Stability of entropy solutions to the Cauchy problem for a class of hyperbolic-parabolic equations, SIAM J. Math. Anal. 33 (2001), 751-762. MR.1884720 (2002m:35125)

[6] G.-Q. Chen and H. Frid, Decay of entropy solutions of nonlinear conservation laws, Arch. Rational Mech. Anal. 146(2) (1999), 95-127. MR1718482(2000h:35099)

[7] G.-Q. Chen and B. Perthame, Well-posedness for nonisotropic degenerate parabolichyperbolic equations, Annales de l'Institut Henri Poincaré Analyse Non Linéaire 20 (2003), 645-668. MR1981403(2004c:35235)

[8] C. M. Dafermos, Hyperbolic Conservation Laws in Continuum Physics, second edition, Springer-Verlag: Berlin, 2005. MR2169977 (2006d:35159)

[9] A.-L. Dalibard and B. Perthame, Existence of solutions of the hyperbolic Keller-Segel model, Trans. Amer. Math. Soc. 361 (2009), 2319-2335.

[10] C. De Lellis, F. Otto, and M. Westdickenberg, Structure of entropy solutions for multidimensional scalar conservation laws, Arch. Rational Mech. Anal. 170 (2003), 137-184. MR2017887 (2005c:35191)

[11] B. Engquist and W. E, Large time behavior and homogenization of solutions of twodimensional conservation laws, Comm. Pure Appl. Math. 46 (1993), 1-26. MR.1193341 (94a:35077)

[12] M. S. Espedal, A. Fasano, and A. Mikelić, Filtration in Porous Media and Industrial Applications, Lecture Notes in Math. 1734, Springer-Verlag: Berlin, 2000. MR1816142(2001i:76096)

[13] F. Golse, P.-L. Lions, B. Perthame, and R. Sentis, Regularity of the moments of the solution of a transport equation, J. Funct. Anal. 76 (1988), 110-125. MR923047 (89a:35179)

[14] P.-E. Jabin, F. Otto, and B. Perthame, Line-energy Ginzburg-Landau models: zero-energy states, Ann. Sc. Norm. Super. Pisa Cl. Sci. (5) 1 (2002), 187-202. MR1994807(2005k:35070) 
[15] P.-E. Jabin and B. Perthame, Regularity in kinetic formulations via averaging lemmas. A tribute to J. L. Lions, ESAIM Control Optim. Calc. Var. 8 (2002), 761-774 (electronic). MR:1932972(2003j:35207)

[16] K. H. Karlsen and N. H. Risebro, Convergence of finite difference schemes for viscous and inviscid conservation laws with rough coefficients, M2AN Math. Model. Numer. Anal. 35(2) (2001), 239-269. MR1825698(2002b:35138)

[17] P.-L. Lions, B. Perthame, and E. Tadmor, A kinetic formulation of multidimensional scalar conservation laws and related equations, J. Amer. Math. Soc. 7 (1994), 169-191. MR1201239 (94d:35100)

[18] A. Michel and J. Vovelle, Entropy formulation for parabolic degenerate equations with general Dirichlet boundary conditions and application to the convergence of FV methods, SIAM J. Numer. Anal. 41 (2003), 2262-2293 (electronic). MR2034615 (2004k:35228)

[19] F. Murat, Compacité par compensation, Ann. Sc. Norm. Sup. Pisa Cl. Sci. (4) 5 (1978), 489-507. MR506997(80h:46043a)

[20] J. Nolen, G. Papanicolaou, and O. Pironneau, A framework for adaptive multiscale methods for elliptic problems, Multiscale Model. Simul. 7 (2008), 171-196. MR.2399542

[21] B. Perthame, Kinetic Formulation of Conservation Laws, Oxford Univ. Press: Oxford, 2002. MR2064166 (2005d:35005)

[22] B. Perthame and P. E. Souganidis, A limiting case for velocity averaging, Ann. Sci. École Norm. Sup. (4) 31 (1998), 591-598. MR 1634024 (99h:82064)

[23] B. Perthame and P. E. Souganidis, Dissipative and entropy solutions to non-isotropic degenerate parabolic balance laws, Arch. Rational Mech. Anal. 170 (2003), 359-370. MR2022136 (2006c:35162a)

[24] D. Serre, Systems of Conservation Laws, Cambridge University Press: Cambridge, 2000. MR:1775057 (2001c:35146)

[25] E. Tadmor and T. Tao, Velocity averaging, kinetic formulations and regularizing effects in quasilinear PDEs, Comm. Pure Appl. Math. 60 (2007), 1488-1521. MR2342955 (2008g:35011)

[26] L. Tartar, Compensated compactness and applications to partial differential equations, In: Research Notes in Mathematics, 39, Nonlinear Analysis and Mechanics: Heriot-Watt Symposium, Vol. 4, ed. R.J. Knops, Pitman Press: Boston-London, 1979. MR.584398 (81m:35014)

School of Mathematical Sciences, Fudan University, Shanghai 200433, People's Republic of China - And - Department of Mathematics, Northwestern University, EVANSTON, IlLiNOIS 60208-2730

E-mail address: gqchen@math.northwestern.edu

Université Pierre et Marie Curie, Université Paris 06, UMR 7598, Laboratoire Jacques-Louis Lions, 4, Place Jussieu, 75005, Paris, France - and - Institut Universitaire de France, 103, BD Saint-Michel, 75005, Paris, France

E-mail address: benoit.perthame@upmc.fr 